\title{
NCSU-SAS-Ning: Candidate Generation and Feature Engineering for Supervised Lexical Normalization
}

\author{
Ning Jin \\ Text Analytics R\&D \\ SAS Institute, Inc. \\ Cary, NC, USA \\ Ning.Jinesas.com
}

\begin{abstract}
User generated content often contains non-standard words that hinder effective automatic text processing. In this paper, we present a system we developed to perform lexical normalization for English Twitter text. It first generates candidates based on past knowledge and a novel string similarity measurement and then selects a candidate using features learned from training data. The system has a constrained mode and an unconstrained mode. The constrained mode participated in the W-NUT noisy English text normalization competition (Baldwin et al., 2015) and achieved the best F1 score.
\end{abstract}

\section{Introduction}

User generated content, such as customer reviews, forum discussions, text messages and Twitter text, is of great value in applications like understanding users, trend discovery and crowdsourcing. For example, by reading the Twitter text posted by a user, a company can learn the user's preferences and connections and use the information for targeted advertising. For another example, by reading Amazon customer reviews about a certain product, a shopper can collect a lot of product information that is not available from manufacturers and retailers. Unfortunately, user generated content often contains ungrammatical sentence structures and nonstandard words, which hinders automated text processing.

In this paper, we present a solution that attempts to perform lexical normalization (Han et al., 2011) for English Twitter text based on train- ing text with human annotation (Baldwin et al., 2015). The solution has a constrained mode and an unconstrained mode. Both modes have the same architecture and components. Both use the annotated training data and CMU's ark POS tagger (Gimpel et al., 2011). The difference between them is parameter settings and the usage of a canonical lexicon dictionary by the unconstrained mode.

This paper is organized as follows: Section 2 describes the architecture and components shared by the constrained and unconstrained modes. Section 3 lists what resources are used by each system. In Section 4, we describe the different settings of the constrained and unconstrained modes and compare their performance. Section 5 concludes the paper and discusses future work.

\section{Architecture and Components of the System}

Given a tokenized English tweet $T=\left(t_{1}, t_{2}, \ldots\right.$, $\left.t_{n}\right)$, where $t_{i}$ is the $i$-th token and $n$ is the total number of tokens, our normalization system processes one token at a time and has two components: candidate generation and candidate evaluation. To normalize token $t_{i}$, the system first generates a small set of candidate canonical forms. Then it calculates a confidence score for each candidate and selects the one with the highest confidence score as the canonical form of token $t_{i}$. How to generate candidates and how to calculate confidence scores are learned from training data.

\subsection{Candidate Generation}

The candidates of a token $t_{i}$ include:

- The token itself 
- All tokens that are considered canonical forms of $t_{i}$ in the training data (static mapping dictionary)

- A split into multiple canonical forms if the token $t_{i}$ is not a canonical form (for example, "loveyourcar" $\rightarrow$ "love your car")

- Top- $m$ most similar canonical forms found in training data (see subsection 2.2 for details of similarity measurement)

Figure 1 shows an example of training data and a new tweet for normalization. Table 1 shows a portion of the static mapping dictionary learned from the training data.

For token "ur" in the new tweet, the token itself is "ur". All of its possible canonical forms present in the training data are "you are" and "your". Let $m=1$, the most similar canonical form is "your". Therefore, the candidates of "ur" include "ur", "you are" and "your". For token "looove" in the new tweet, the token itself is "looove". It is absent in the training data, so it does not have its own canonical form available as candidates. Among all the canonical forms present in training data, canonical form "love" is most similar to "looove". Therefore, the candidates of "looove" include "looove" and "love".

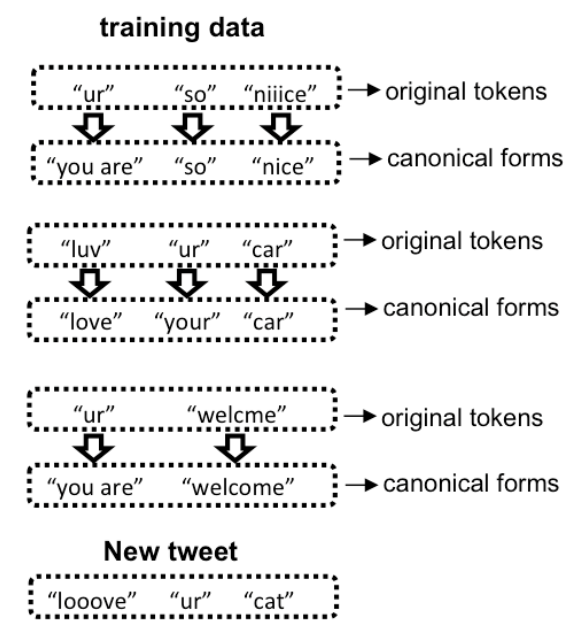

Figure 1: An Example of Training Data and a New Tweet for Normalization

\begin{tabular}{|l|l|}
\hline Key (token) & Value (canonical forms) \\
\hline "ur" & "your", "you are" \\
\hline "so" & "so" \\
\hline "niiice" & "nice" \\
\hline "luv" & "love" \\
\hline "car" & "car" \\
\hline "welcme" & "welcome" \\
\hline
\end{tabular}

Table 1: Static Mapping Dictionary Learned from Training Data

\subsection{Similarity Index}

We measure similarity between two strings by first representing each string with a set of similarity features and then evaluating similarity with Jaccard Index (Levandowsky et al., 1971) of the two similarity feature sets.

The similarity features of a string $s$ include $n$ grams and $k$-skip- $n$-grams in $s$. In this paper, an $n$-gram in string $s$ is defined as a contiguous sequence of $n$ characters in $s$. A $k$-skip- $n$-gram in string $s$ is a generalization of $n$-gram with gaps between characters and is defined as a sequence of $n$ characters where the maximum distance between two characters is $k$. We prepend (append) a "\$" to $n$-grams that appear at the beginning (end) of the string. We use "|" to indicate gaps in skip-grams. For example, Table 2 shows the similarity feature sets of "love", "looove", "car" and "cat", with $n=2$ and $k=1$.

\begin{tabular}{|l|l|}
\hline String & Similarity Feature Set \\
\hline "love" & "\$lo", "ov", "ve\$", "l|v", "ole" \\
\hline "looove" & "\$lo", "oo", "ov", "ve\$", "l|o", "o|o", "o|v", "o|e" \\
\hline "car" & "\$ca", "ar\$", "c|r" \\
\hline "cat" & "\$ca", "at\$", "c|t" \\
\hline
\end{tabular}

Table 2: An Example of Similarity Features $(n=2, k=1)$

Let the similarity feature set of a string $s$ be $f(s)$, then we measure string similarity between $s_{1}$ and $s_{2}$ by:

$$
\begin{aligned}
\operatorname{similarity}\left(s_{1},\right. & \left.s_{2}\right) \\
& =J \operatorname{JaccardIndex}\left(f\left(s_{1}\right), f\left(s_{2}\right)\right) \\
& =\frac{\left|f\left(s_{1}\right) \cap f\left(s_{2}\right)\right|}{\left|f\left(s_{1}\right) \cup f\left(s_{2}\right)\right|}
\end{aligned}
$$

For example, in Table 2, "love" and "looove" share similarity features " "\$1o", "ov", "ve\$", "o|e" $\}$. The union of their similarity feature sets is \{ "\$10", "oo", “ov", "ve\$", "l|v", "l|o", "o|o", "o|v", "o|e"\}. The similarity score between "love" and "looove" is $4 / 9=0.44$.

Different weights can be assigned to different similarity features when calculating similarity scores because $n$-grams at different positions have different importance for word recognition (White et al., 2008). For example, in the example shown in Table 2, we can assign weight 3 to bigrams at the beginning and end of strings and weight 1 to other features, and then the similarity score between "love" and "looove" becomes $8 / 13=0.615$.

The similarity feature set calculation can use multiple $(n, k)$ configurations instead of just one. For example, the similarity feature set can be composed of bigrams, trigrams, 1-skip-bigrams 
and 2-skip-bigrams together. If $k=0$, it means no skip-gram is used.

This similarity measurement penalizes text edits such as insertion, deletion and substitution. Compared with Levenshtein distance (Levenshtein, 1966), one disadvantage of our similarity measurement is that two different strings may have 1.0 similarity score because the similarity feature set can only capture local character order information. For example, strings "aaabaa" and "aaaabaa" have exactly the same similarity feature set \{“\$aa”, “ab”, “ba”, “aa\$”, “a|a”, “a|b", "b|a" $\mid$ and thus have 1.0 similarity score. Including skip-gram and using a larger $n$ in similarity feature calculation can mitigate this problem but cannot prevent it. Fortunately, this should be very rare when the similarity measurement is applied to two real world twitter tokens because such cases require the strings to be long and contain repetitive $n$-grams and skip-grams. One advantage of our similarity measurement over Levenshtein distance is that it takes into account the string length when penalizing text edits. The same text edit has a bigger impact when it occurs in a short string than in a long string because of the denominator in Jaccard Index. Another advantage of our similarity measurement is that it better handles repetition characters, which is commonly used in Twitter. For example, for our similarity measurement, both "looove" and "loooooove" are equally similar to "love". For Levenshtein distance, "loooooove" takes a much heavier penalty than "looove". ${ }^{1}$ The biggest advantage of our similarity measurement over Levenshtein distance is the lower computational complexity. Let the length of a string $s$ be $l(s)$. The feature set size of $s$ is bounded by $O(l(s))$. Then the complexity of calculating Levenshtein distance between $s_{1}$ and $s_{2}$ is $O\left(l\left(s_{1}\right) l\left(s_{2}\right)\right)$, which is quadratic when two strings have similar length. On the contrary, the complexity of calculating our similarity measurement is $O\left(l\left(s_{1}\right)+l\left(s_{2}\right)\right)$, which is linear. ${ }^{2}$

We index all the canonical forms in the training data based on similarity features to facilitate

\footnotetext{
${ }^{1}$ Certain preprocessing can mitigate this problem for Levenshtein distance. For example, all single character repetitions get reduced to two before Levenshtein distance is calculated. But it does not handle repetition of multiple characters, e.g. "lolol".

${ }^{2}$ The linear complexity depends on using hash table to calculate set union and intersection. Another implementation is sorting the similarity features first and then calculating union and intersection, which has $O\left(l^{*} \log (l)\right)$ complexity $(l$ is the longer string length of the two strings) and is still better than quadratic complexity of Levenshtein distance.
}

finding top- $m$ canonical forms that are most similar to the query token. Given a query token, we can quickly narrow down our search space to canonical forms that share at least one similarity feature with the query token. Further efficiency improvement can be achieved by approximating the denominator in Jaccard Index based on string lengths or by imposing restrictions on the minimum number of similarity features to be shared by query token and results.

\subsection{Candidate Evaluation}

Given a tweet $T$, one of its token $t_{i}$ and one of the token's candidate $c$, we train a binary classifier that predicts whether $c$ is the correct canonical form of $t_{i}$ in the tweet $T$ and outputs a confidence score for the prediction. Among the candidates that the classifier predicts to be the correct canonical forms, we select the one with the highest confidence score as the canonical form of $t_{i}$. In our implementation of the system, we used a random forest classifier (Breiman, 2001) mainly because its training speed is faster and its performance is relatively insensitive to parameter values, but other binary classification algorithm should also work.

This step is mostly feature engineering and we used the following features:

\section{- Support and confidence}

We calculate the support of token $t_{i}$ (number of times $t_{i}$ appears) and confidence of token $t_{i}$ being normalized to candidate $c$ (percentage of times $t_{i}$ is normalized to $c$ ) according to training data and use them as features for classification. For example, in the training data shown above, the support of token "ur" is 3 and the confidence of normalizing "ur" to "you are" is $2 / 3=0.67$. The confidence of normalizing "ur" to "your" is $1 / 3=0.33$. If the token $t_{i}$ is absent in the training data, e.g. "looove", then the support and confidence are both zero. If the token $t_{i}$ is present but the normalization from $t_{i}$ to $c$ is absent in training data, then only the confidence is zero. These features are context free and the intuition is that the higher the support and confidence are (high support is necessary in case of small sample), the more likely that $c$ is the correct canonical form of $t_{i}$.

\section{- String information}

We calculate the string similarity score (Jaccard Index of feature sets) between token $t_{i}$ and candidate $c$ and use it as a feature for 
classification. String similarity score is a good feature for difference between token and its canonical form caused by misspelling (for example, "seperate" $\rightarrow$ "separate"), but it is not a good feature for difference caused by abbreviation (for example, "lol" $\rightarrow$ "laughing out loud"). Therefore, we also add string length and difference in string length between $t_{i}$ and $c$ so that classifier can choose to ignore string similarity score when necessary.

All string information features are context free.

\section{- POS tagging information}

One of the motivations of text normalization is to facilitate subsequent tasks, such as partof-speech tagging and named entity recognition. Therefore, good text normalization should make the subsequent tasks easier. We observed that in the training data, in $90 \%$ of the cases where a token is normalized to another token, the canonical form has higher POS tagging confidence, based on the ark POS tagger (Gimpel et al., 2011), than the original. Therefore we use change in POS tagging confidence at position $i$ in tweet $T$ before and after normalizing $t_{i}$ to $c$ as a feature for classification.

We also include change in mean POS tagging confidence in tweet $T$ because changing one token can affect the confidence of tagging other tokens. In addition to change in POS tagging confidence, we use POS tags of tokens $t_{i-1}$ and $t_{i}$ as features (tag is empty if $t_{i}$ is the first token) because there can be patterns of consecutive POS tags and some patterns are much more frequent than others.

All POS tagging features use context information.

The importance of these classification features are evaluated in Section 4.

To train the classifier, we generate candidates for each token in training data and label each pair according to human annotation. If the candidate is the correct canonical form of the token in the tweet, then the pair is labeled as class 1; otherwise the pair is labeled as class 0 . Feature vectors with features described above are calculated for each pair. Then a random forest binary classifier is learned. When the classifier is learned, the class (label) weights are adjusted inversely proportional to class frequencies in the data because the data is imbalanced and majority of the observations are in class 0 .

\section{Resources Employed}

We implemented two modes for our normalization system: a constrained mode and an unconstrained mode. ${ }^{3}$ The constrained mode uses only the training data train_data_20150430.json and the ark twitter POS tagger (Gimpel et al., 2011). The unconstrained mode uses the canonical English lexicon dictionary scowl.american.70, in addition to all resources used by the constrained mode.

\section{Settings and Evaluation}

For both the constrained and unconstrained modes, we use only bigrams and 1-skip-bigrams as similarity features. The differences between the two modes are listed below.

For the constrained mode:

- It uses best-scoring canonical forms from the similarity index as candidates.

- It uses similarity index for candidate generation only when the token contains repetitive characters (same character occupying consecutive positions). ${ }^{4}$

- It builds a similarity index based on all canonical forms present in the training data.

- Dictionary and feature learning and classifier training are based on the same data set.

For the Unconstrained mode:

- It uses top-3 best-scoring canonical forms from the similarity index as candidates.

- It builds a similarity index based on all canonical forms in the training data and all lexicons in the dictionary scowl.american.70.

- It always uses the similarity index for candidate generation.

\footnotetext{
${ }^{3}$ The unconstrained mode was developed when we were writing this paper, after the annotation for the test data set was revealed. Only the constrained mode was submitted for the competition.

${ }^{4}$ This is because a similarity index based on smaller vocabulary leads to less reliable candidates. For example, in the example shown in Figure 1, the similarity index returns "car" as a candidate of "cat" because "car" is the most similar canonical form in training data. In a larger vocabulary, "cat" itself should be the most similar canonical form.
} 
- Dictionary and feature learning and classifier training are based on different data sets.

For the constrained mode, dictionaries (including static mapping dictionary and similarity index), classification feature calculation and classifier training are based on the same data set. It causes overfitting because the dictionaries and the support and confidence features leak label information. However, our cross-validation results show that learning dictionaries, support and confidence features, and classifier on the same data set generates better generalization as well. It leads to better F1 score than splitting the data set into two parts and learning dictionaries and features on one part and learning the classifier on the other part. This is because having large dictionaries is crucial for candidate generation and the correct canonical form cannot be found if it is not among the candidates. Using all the available data instead of splitting it allows the system to learn larger dictionaries and more than makes up for the overfitting problem.

For the unconstrained mode, dictionaries and features are learned on $67 \%$ of the available data and the classifier is learned on $33 \%$ of the available data (random split). This is different from constrained mode because the unconstrained mode already has a very large canonical form dictionary in scowl.american.70 and the accuracy of selecting the correct canonical form becomes the bottleneck.

We used the data sets provided by the WNUT 2015 lexical normalization competition (described in (Baldwin et al., 2015)) for evaluation. During our development of the systems, only the training data file train_data_20150430.json was used for any parameter selection and design decisions. We used cross-validation to estimate system performance. The constrained and unconstrained modes have separate classifier training.

Table 3 shows the performance of the constrained mode with different sets of classification features based on the test data file test_truth.json concealed from development. It can be seen that the support and confidence features are the most important for achieving high F1 score. Without the support and confidence features, the F1 score of the constrained mode decreases by 0.0521 . The POS tagging features constitute the second most important feature set. Without POS tagging features, the F1 score goes down by 0.0129 . The string features are the least important set of fea- tures as they lead to very marginal improvement in F1 score.

\begin{tabular}{|l|l|l|l|}
\hline & Precision & Recall & F1 Score \\
\hline Constrained w/ all features & 0.9061 & 0.7865 & 0.8421 \\
\hline $\begin{array}{l}\text { Constrained w/o support and } \\
\text { confidence features }\end{array}$ & 0.9423 & 0.6803 & 0.7901 \\
\hline $\begin{array}{l}\text { Constrained w/o POS tag- } \\
\text { ging features }\end{array}$ & 0.902 & 0.7673 & 0.8292 \\
\hline $\begin{array}{l}\text { Constrained w/o string fea- } \\
\text { tures }\end{array}$ & 0.9102 & 0.7825 & 0.8416 \\
\hline
\end{tabular}

Table 3: Importance of Classification Features

In Table 4, we report the evaluation results based on the test data file test_truth.json concealed from development. For constrained mode, we list the top-two results by teams NCSU_SAS_NING (Ning.cm) and NCSU_SAS_WOOKHEE (Wookhee.cm). For unconstrained mode, we list the top result by team IHS_RD (IHS_RD.um) and the result by our own unconstrained mode (Ning.um), which was developed after the competition ended.

\begin{tabular}{|l|l|l|l|l|}
\hline \multirow{2}{*}{$\begin{array}{l}\text { Perfor- } \\
\text { mance }\end{array}$} & \multicolumn{2}{|l|}{ Constrained Mode } & \multicolumn{2}{l|}{ Unconstrained Mode } \\
\cline { 2 - 5 } & Ning.cm & Wookhee.cm & Ning.um & IHS_RD.um \\
\hline Precision & 0.9061 & 0.9136 & $\mathbf{0 . 9 3 3 9}$ & 0.8469 \\
\hline Recall & 0.7865 & 0.7398 & 0.7582 & $\mathbf{0 . 8 0 8 3}$ \\
\hline F1 Score & $\mathbf{0 . 8 4 2 1}$ & 0.8175 & 0.837 & 0.8272 \\
\hline
\end{tabular}

Table 4: Competition Evaluation Results

It can be seen that our normalization system has the best F1 score in both constrained mode and unconstrained mode. In fact, our constrained mode has the best F1 score overall, better than our unconstrained mode, which seems counterintuitive. Besides, the unconstrained mode is expected to achieve higher recall than the constrained mode because of its much larger dictionary, but the evaluation results show that the unconstrained mode has lower recall and higher precision than the constrained mode. The following three factors lead to the inferior F1 score and recall by our unconstrained mode:

The much larger canonical form dictionary used by the unconstrained mode contains many rarely used words and having such words as candidates causes the candidate evaluation component to be more conservative in selecting candidates other than the original tokens (higher precision and lower recall). A potential solution is to use a smaller dictionary of most frequently used words instead of a large dictionary or to use a dictionary with word frequency based on a large corpus.

Even if we exclude the rare words, the mere increase in number of candidates per token makes selecting the correct candidate more challenging. For example, our unconstrained mode 
successfully suggests "Brooklyn" as a candidate for token "Brklyn", which our constrained mode is incapable of, but the candidate evaluation component fails to select "Brooklyn" as the correct canonical form. A potential solution is to include more context information for candidate evaluation. For example, text likelihood estimated by a CRF model before and after normalization can be added as classification features. Having word frequency as a feature can also be helpful.

The binary class labeling in the candidate evaluation component does not differentiate normalization without change (e.g. "car" $\rightarrow$ "car") from normalization with change (e.g. "ur" $\rightarrow$ "your"). As a result, we are unable to tune parameters to favor normalization with change in order to achieve a better trade-off between precision and recall (higher recall and slightly lower precision), which means higher F1 score. A potential solution is to change the candidate evaluation component into a two-level classification. The first level classifies whether the normalization needs any change. If no, then the token itself is output as the normalization result. If yes, then the second level classification assigns a confidence score to each candidate that is different from the token and outputs the one with the highest score as the result.

\section{Conclusions and Future Work}

In this paper, we present a system to perform lexical normalization for English Twitter text, with a constrained mode and an unconstrained mode. Our constrained mode achieves the top F1 score in the W-NUT noisy text normalization competition and outperforms other participants' unconstrained modes. Our unconstrained mode currently has slightly lower recall and F1 score than the constrained mode, but it has a lot more room for improvement as discussed in the evaluation section. Future work includes implementing the ideas to improve the unconstrained mode and exploring semi-supervised and unsupervised text normalization. One potential solution for unsupervised text normalization is first clustering tokens based on context (e.g. Brown clustering (Brown et al., 1992)) and then choosing the most frequent token in each cluster as the canonical form for all tokens in that cluster.

\section{Reference}

T. Baldwin, M. Catherine, B. Han, Y.B. Kim, A. Ritter and W. Xu. 2015. Shared Tasks of the 2015
Workshop on Noisy User-generated Text: Twitter Lexical Normalization and Named Entity Recognition. In Proc. of WNUT.

L. Breiman. 2001. Random Forests. Machine Learning, 45(1), 5-32.

P. Brown, P. deSouza, R. Mercer, V. Della Pietra, J. Lai. 1992. Class-Based n-gram Models of Natural Language. Computational Linguistics, vol. 18, pp. 467-479.

K. Gimpel, N. Schneider, B. O'Connor, D. Das, D. Mills, J. Eisenstein, M. Heilman, D. Yogatama, J. Flanigan, and N. A. Smith. 2011. Part-of-speech tagging for Twitter: Annotation, features, and experiments. In Proc. of ACL.

B. Han and T. Baldwin. 2011. "Lexical normalisation of short text messages: Makn sens a \#twitter". In Proc. of ACL.

M. Levandowsky and D. Winter. 1971. Distance between sets. Nature 234 (5): 34-35.

V. Levenshtein. 1966. Binary codes capable of correcting deletions, insertions, and reversals. Soviet Physics Doklady 10 (8): 707-710.

S. White, R. Johnson, S. Liversedge, K. Rayner. 2008. Eye Movements When Reading Transposed Text: The Importance of Word-Beginning Letters. Journal of experimental psychology Human perception and performance. 